\title{
Comparación de la comunidad de coleópteros acuáticos (Adephaga y Polyphaga) en dos cuencas hidrográficas con distinto grado de acción antropogénica (Pontevedra, NO de España)
}

\author{
Cesar João Benetti*, Ana Isabel Alonso y Josefina Garrido \\ Departamento de Ecología y Biología Animal, Facultad de Biología, Universidad de Vigo, 36200, Vigo (España) \\ e-mail: jgarrido@uvigo.es.
}

* Autor responsable de la correspondencia: cjbenetti@uvigo.es

\begin{abstract}
RESUMEN
Comparación de la comunidad de coleópteros acuáticos (Adephaga y Polyphaga) en dos cuencas hidrográficas con distinto grado de acción antropogénica (Pontevedra, NO de España)

En este trabajo se estudia la fauna de coleópteros acuáticos (Adephaga y Polyphaga) de las cuencas hidrográficas de los ríos Lagares y Miñor (Pontevedra, NO de España). Se muestrearon 20 puntos en cuatro campañas estacionales (mayo 2001-enero 2002). Se estudiaron un total de 1597 individuos, identificándose 35 especies de las familias Haliplidae, Gyrinidae, Dytiscidae, Helophoridae, Hydrochidae, Hydrophilidae, Hydraenidae, Elmidae, Dryopidae y Scirtidae. Se citan por primera vez para la provincia de Pontevedra la familia Hydrochidae (Hydrochus angustatus) los géneros Esolus (Esolus parallelepipedus) y Megasternum (Megasternum concinnum) y las especies Helophorus flavipes, Elmis aenea y Elmis maugetii maugetii. La variación espacial y temporal de la riqueza, abundancia y diversidad han sido analizadas, así como la afinidad entre los puntos de muestreo, basada en el análisis de la composición faunística. Los resultados indican que las comunidades típicas de aguas no contaminadas quedan claramente separadas de las comunidades de aguas contaminadas, indicando este fenómeno la influencia negativa de la contaminación sobre la fauna.
\end{abstract}

Palabras clave: Coleópteros acuáticos, acción antropogénica, diversidad, Ríos Lagares y Miñor, Galicia.

\begin{abstract}
Comparison of the community of aquatic Coleoptera (Adephaga and Polyphaga) of two hydrographic river basins with different degree of anthropic action (Pontevedra, $N W$ of Spain)

In this work we studied the fauna of aquatic Coleoptera (Adephaga and Polyphaga) of the hydrographic river basins of the Lagares and Miñor Rivers (Pontevedra, NW of Spain). Twenty sites were sampled in four seasonal campaigns between May of 2001 and January of 2002. A total of 1,597 specimens belonging to 35 species of the families Haliplidae, Gyrinidae, Dytiscidae, Helophoridae, Hydrochidae, Hydrophilidae, Hydraenidae, Elmidae, Dryopidae and Scirtidae were collected. The family Hydrochidae (Hydrochus angustatus), the genus Esolus (Esolus parallelepipedus), and Megasternum (Megasternum concinnum), and the species Helophorus flavipes, Elmis aenea, and Elmis maugetii maugetii are all new records for the province. Spatial and seasonal variations in richness, abundance and diversity were analysed, in addition the similarity between sites was also analysed based on the total fauna composition of each. The results indicate that the typical communities of non-polluted waters are separated from those of polluted waters, indicating the negative influence of the contamination on the fauna.
\end{abstract}

Keywords: Aquatic Coleoptera, anthropic action, diversity, Lagares and Miñor Rivers, Galicia. 


\section{INTRODUCCIÓN}

Los ecosistemas fluviales han estado sometidos a profundos cambios como consecuencia de perturbaciones de origen antropogénico, debido principalmente al aumento de la población humana y consiguiente degradación de los recursos naturales. La protección de estos ecosistemas es fundamental para la conservación de su biodiversidad, ya que con la alteración de su estructura y contaminación del agua, los ríos han perdido gran parte de la diversidad biológica. En ese contexto, y entre los macroinvertebrados de las aguas continentales, los coleópteros están entre la fauna mejor representada en cuanto a riqueza de especies y muchos de ellos pueden ser utilizados como indicadores de la calidad del agua de esos hábitats. (Castella et al., 1984; Flechtner, 1986; Foster et al., 1990, 1992; Ribera \& Foster 1993; Eyre et al., 1993; Sánchez et al., 2003; 2004; Abellán et al., 2005).

Estudios faunísticos y taxonómicos sobre el grupo de coleópteros acuáticos han sido relativamente abundantes en la península Ibérica, especialmente en los últimos años (Sainz-Cantero \& Alba-Tercedor, 1991a, 1991b; Millán et al, 1992; 1993; 1997; 2001a; 2001b; 2002; 2005; Ribera \& Aguilera, 1995; Valladares et al., 2000; Ribera et al., 1995a; 1995b; Rico, 1996; Sáinz-Cantero \& Cortés-Romero, 1996; Garrido et al., 1994; 1996, 1997; Régil Cueto \& Garrido, 1998, Rico \& García-Aviles, 1998; Castro et al., 2003; Garrido \& Gayoso, 2005). En el noroeste peninsular también, se pueden citar una serie de estudios, que permiten dar a conocer este grupo faunístico de una manera importante (González \& Novoa, 1988, 1995; Garrido \& Régil, 1994; Gayoso et al., 1997; Garrido \& Sáinz-Cantero, 2004; González et al, 2005).

Relaciones entre comunidades de coleópteros acuáticos y el medio que habitan, han sido contempladas en distintos estudios (Puig, 1983; García Criado \& Fernández-Aláez, 1994; Gallardo et al., 1995; Millán et al, 1996; Moreno et al., 1997; Lozano-Quilis et al., 2001; Valladares et al., 2002; Muñoz, 2003; González et al., 2003; Argerich et al, 2004; Guerrero et al, 2004).
Otros recogen aspectos relativos a la autoecología (Sáinz-Cantero et al., 1987) y fenología de las especies (Valladares et al., 1994; Valladares \& Garrido, 2001).

Menos frecuentes son los trabajos que estudian los efectos de la contaminación sobre la fauna de coleópteros acuáticos. Entre ellos son destacables los estudios de la influencia de la contaminación minera sobre la fauna de Elmidae e Hydraenidae (García-Criado, 1999; 2002; García-Criado \& Fernández-Aláez, 1995; 2001; García-Criado et al., 1995; 1999). En este sentido, en este trabajo se estudia el efecto de la contaminación antropogénica sobre la comunidad de coleópteros acuáticos de dos cuencas hidrográficas, muy cercanas entre sí en la provincia de Pontevedra. Entre otros aspectos, se analiza la diversidad espacial y temporal y la afinidad faunística entre las dos cuencas que están afectadas de forma distinta por la contaminación.

\section{ÁREA DE ESTUDIO}

Las cuencas hidrográficas de los ríos Lagares y Miñor están localizadas en el suroeste de la provincia de Pontevedra, entre la ría de Vigo y la desembocadura del río Miño; abarcan las coordenadas $8^{\circ} 38^{\prime}$ y $8^{\circ} 50^{\prime}$ de longitud oeste y $42^{\circ} 8^{\prime}$ y $42^{\circ} 14^{\prime}$ de latitud norte (Fig. 1).

El río Lagares nace en las proximidades del aeropuerto de Peinador a 300 metros de altitud, estando ubicado en el municipio de Vigo y la mayor parte de sus $20 \mathrm{~km}$ de longitud discurren por el casco urbano de la ciudad. Gran parte de su curso original se presenta alterado, por los diferentes encauzamientos a que se ha visto sometido. El río Miñor nace en las estribaciones de la Serra do Galiñeiro, a 400 metros de altitud y está ubicado en los municipios de Vigo, Gondomar, Nigran y Baiona. El río Miñor, con algo más de $20 \mathrm{~km}$ de longitud, discurre tanto por áreas rurales como por áreas urbanas y su cauce se presenta, por lo general, poco alterado. Los dos ríos discurren en sentido E-W y sus cuencas están separadas por una serie de montes de altura no superior a 400 metros, hasta su desembocadura en la Ría de Vigo. 


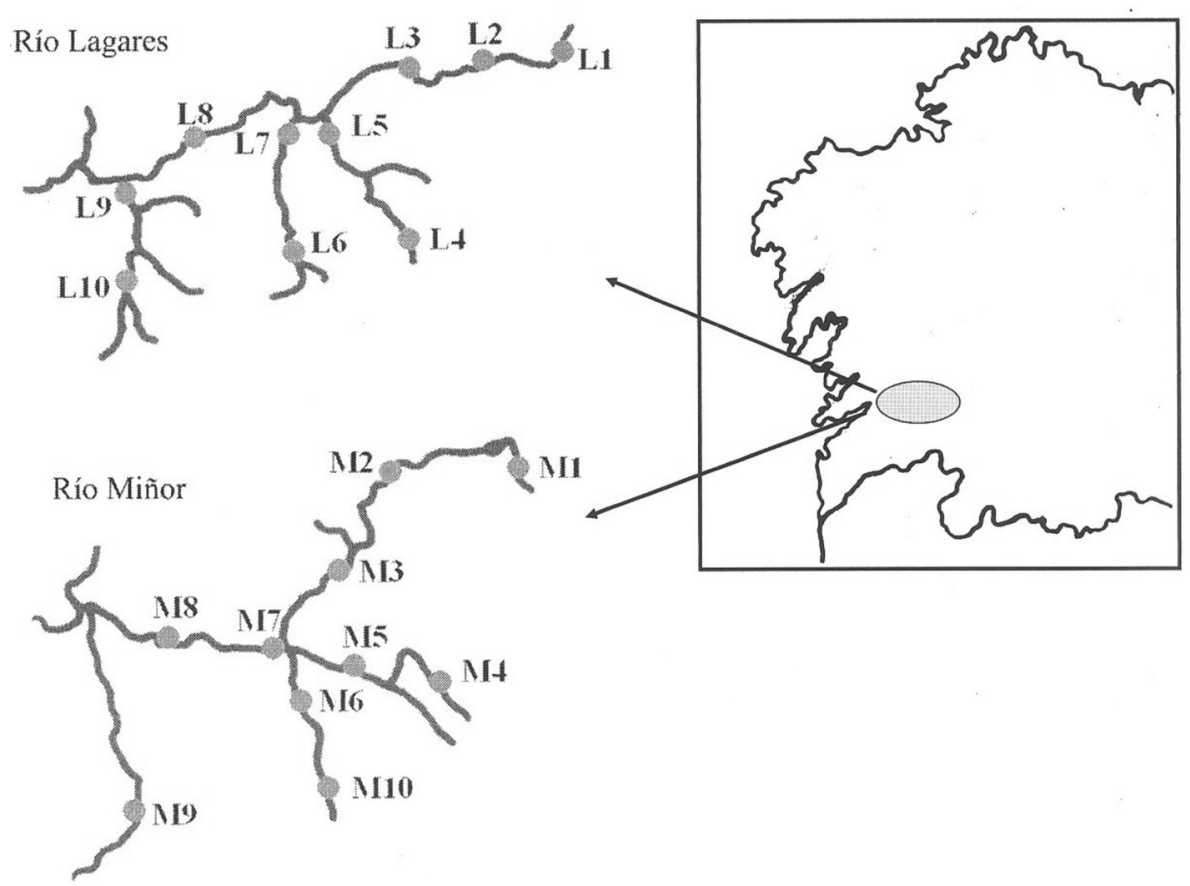

Figura 1. Localización de los ríos Lagares y Miñor en la Península Ibérica y sus respectivos puntos de muestreo. Location of the Lagares and Miñor rivers in the Iberian peninsula and the sampling sites on each.

El clima de esta zona, mediterráneo húmedo, se caracteriza por las abundantes precipitaciones (entre 1500 y $1800 \mathrm{~mm}$ anuales), temperaturas suaves (14-15 ${ }^{\circ} \mathrm{C}$ media/año) y por el aumento de la temperatura y aridez en verano. Geológicamente, es un área de transición entre zonas graníticas (Granitoides hercínicos) y metamórficas (Galicia-Tras os Montes). Las comarcas por las que discurren ambos ríos están caracterizadas, en mayor o menor grado, por su elevada urbanización, con una densidad media superior a 500 habitantes por $\mathrm{km}^{2}$ (Rodríguez Iglesias, 1998).

\section{MATERIAL Y MÉTODOS}

Las muestras se tomaron en 20 puntos, 10 en cada una de las cuencas hidrográficas, designados como L1... L10 para los puntos muestreados en la cuenca del río Lagares y M1... M10 para los muestreados en la cuenca del río Miñor (Tabla 1). Los muestreos se realizaron durante un ciclo anual (mayo 2001 a enero 2002) y en las cuatro campañas estacionales (primavera, verano, otoño e invierno), mediante un programa estandarizado, estableciendo un transecto de cinco metros de longitud a lo largo del curso, independiente del tipo de hábitat. Para la captura de los ejemplares se utilizó una red entomológica acuática de $30 \mathrm{~cm}$ de diámetro, $60 \mathrm{~cm}$ de fondo y malla de $0.1 \mathrm{~mm}$.

Para la selección de los puntos de muestreo se tuvo en cuenta el cauce principal y sus afluentes. De esta forma se realizaron muestreos en las cabeceras, tramos medios y bajos de los ríos; de forma que se pudieran detectar las posibles alteraciones provocadas por la acción antropogénica. En ese sentido fueron establecidos distintos niveles de alteración según el grado de acción antropogénica, en función de las actividades desarrolladas en los márgenes y proximidades. Así, se consideraron de impacto leve los puntos con influencia de actividades agrícolas poco contaminantes, sin llegar a utilizar pesticidas; moderado para los puntos que presentan actividades 
Tabla 1. Código y nombre de los puntos (L: Lagares; M, Miñor) y el grado de impacto antropogénico atribuido. Code and name of sites (L: Lagares sites; M, Miñor sites) and anthropic impact attributed.

\begin{tabular}{ccc}
\hline Código & Nombre & Nivel de impacto \\
\hline L1 & Aeropuerto & moderado \\
\cline { 2 - 2 } L2 & Rans & moderado \\
\cline { 2 - 2 } L3 & Sello & moderado \\
L4 & San Cribán & leve \\
\cline { 2 - 2 } L5 & Sabu & moderado \\
L6 & Beade & leve \\
L7 & Seur & moderado \\
L8 & San Andrés & intenso \\
\cline { 2 - 3 } L9 & Rial & moderado \\
L10 & Fragoselo & leve \\
M1 & Zamans & leve \\
M2 & Outeiro & leve \\
M3 & Pego Negro & leve \\
M4 & Campo Grande & leve \\
M5 & Morgadans & leve \\
M6 & Enxertos & moderado \\
M7 & Gondomar & moderado \\
M8 & Covelas & leve \\
M9 & Sequiña & leve \\
M10 & Remedios Costa & leve \\
\hline & & \\
\hline
\end{tabular}

agrícolas contaminantes por el uso de pesticidas y actividad industrial leve (pequeñas fabricas poco contaminantes); e intenso para los puntos que están sometidos a actividad industrial fuerte (industria pesada). En la Tabla 1 se pueden observar los distintos puntos y su clasificación según el impacto producido por la actividad antropogénica.

"In situ" se midieron los parámetros ambientales de temperatura del agua y del aire, oxigeno disuelto, $\mathrm{pH}$, conductividad y SST (sólidos en suspensión totales). Los ejemplares capturados se fijaron en formaldehído al $4 \%$ y tras su separación y estudio fueron conservados en alcohol de $70^{\circ}$. Los especimenes obtenidos están depositados en la colección científica del laboratorio de entomología de la Universidad de Vigo.

En cuanto a su frecuencia, las especies fueron clasificadas de acuerdo con su presencia en el total de muestreos en 4 categorías: esporádicas o raras (E) con menos del $5 \%$ de frecuencia; poco frecuentes (PF) con una frecuencia del 5 al $15 \%$; habituales $(\mathrm{H})$ del 15 al $40 \%$ y muy frecuentes
(MF) con una frecuencia de aparición superior al $40 \%$. Se han empleado tres parámetros para valorar la estructura de la comunidad: la riqueza específica, la abundancia total y la diversidad, esta última calculada por medio del índice de Shannon-Wiener. La afinidad entre los puntos ha sido analizada basándose en la composición faunística total de cada uno. El dendrograma ha sido construido por un análisis de Cluster (UPGMA), utilizándose el índice de Jaccard. La matriz de datos se construyó con la presencia o ausencia de las especies en cada punto. Para el procesamiento de los datos se utilizó el programa SPSS 12.0 para Windows.

\section{RESULTADOS \\ Composición de la comunidad y notas biogeográficas}

Se estudiaron 1597 individuos, entre larvas y adultos, de 35 especies pertenecientes a las familias Haliplidae, Gyrinidae, Dytiscidae, Helophoridae, Hydrochidae, Hydrophilidae, Hydraenidae, Elmidae, Dryopidae y Scirtidae (Tabla 2).

Mediante la evaluación de la frecuencia de las especies, estas se clasificaron de acuerdo con su presencia en el total de muestreos. En la Tabla 2 se puede observar que la mayoría de las especies presentaron una frecuencia baja o muy baja (especies esporádicas/raras o poco frecuentes), representando el $80 \%$ (28 especies) del total. Las especies habituales (frecuentes) representaron el $17 \%$ (6 especies), y las muy frecuentes solamente alcanzaron el $3 \%$, estando representado este último grupo por una única especie: Limnius volckmari (Panzer, 1793).

La mayoría de estas especies tienen una amplia distribución de tipo Paleártico. Sin embargo, hay que destacar la presencia en estas cuencas hidrográficas de 13 especies endémicas de la península ibérica, lo que corresponde a un $37 \%$ del total. Estos endemismos corresponden a las especies Nebrioporus (Nebrioporus) carinatus (Aubé, 1838) y Stictotarsus bertrandi (Legros, 1956) entre los Dytiscidae. En la familia Hydraenidae están las especies: Hydraena (Hydraena) 


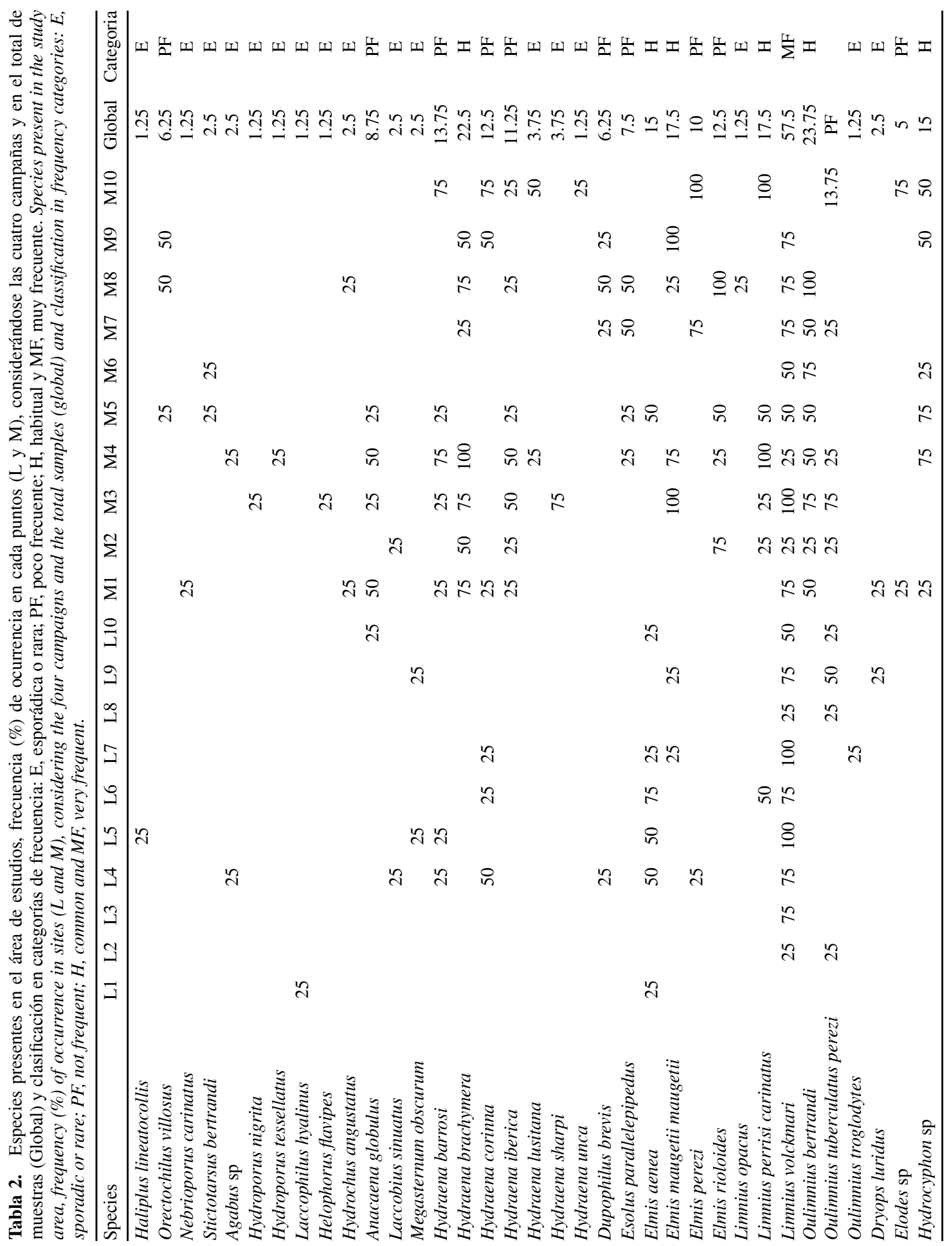


barrosi D'Orchymont, 1934, Hydraena (Hydraena) brachymera d'Orchymont, 1936, Hydraena (Hydraena) corinna d'Orchymont, 1936, Hydraena (H.) iberica d'Orchymont, 1936, Hydraena (H.) lusitana Berthélemy, 1977, Hydraena (Hydraena) sharpi Rey, 1866, Hydraena (Hydraena) unca Valladares, 1989. En la familia Elmidae, son 4 especies endémicas las presentes: Elmis perezi Heyden, 1870, Limnius perrisi carinatus (Pérez-Arcas, 1865), Oulimnius bertrandi Berthélemy, 1964 y Oulimnius tuberculatus perezi (Sharp, 1872) (Ribera et al., 1998). También es notorio destacar que en este trabajo se citan por primera vez para la provincia de Pontevedra la familia Hydrochidae, con la especie Hydrochus angustatus angustatus Germar, 1824;el género Esolus, con la especie Esolus parallelepipedus (Müller, 1806) y el género Megasternum, con la especie Megasternum concinnum (Marsham 1802); además de las especies Helophorus (Rhopalhelophorus) flavipes Fabricius, 1792, Elmis aenea (Müller, 1806) y Elmis maugetii maugetii Latreille, 1798.

Tabla 3. Valores de las variables ambientales medidas "in situ". Values of the environmental variables measured "in situ".

\begin{tabular}{lcccccc}
\hline Muestras & $\mathrm{T}^{\circ}$ aire & $\mathrm{T}^{\circ}$ agua & $\mathrm{pH}$ & $\mathrm{O}_{2}(\mathrm{mg} / \mathrm{l})$ & conductividad & $\mathrm{SST}$ \\
\hline L1O & 14 & 14.7 & 7.25 & 8.1 & 81 & 33 \\
L1P & 22 & 16.6 & 6.49 & 7.2 & 109 & 44 \\
L1V & 22 & 20.9 & 6.45 & 3.9 & 160 & 63 \\
L1I & 8 & 10.6 & 6.49 & 6.66 & 138.6 & 57 \\
L2O & 10 & 14 & 6.49 & 9.7 & 134 & 54 \\
L2P & 21 & 16.4 & 6.68 & 9 & 115 & 46 \\
L2V & 22 & 18.9 & 6.62 & 8.7 & 48 & 121 \\
L2I & 9.5 & 10.9 & 6.6 & 9.21 & 134 & 55 \\
L3O & 16 & 15.2 & 7.5 & 9.96 & 100 & 40 \\
L3P & 19 & 16 & 6.95 & 9.4 & 130 & 52 \\
L3V & 26 & 17.9 & 7.01 & 9.7 & 148 & 59 \\
L3I & 14 & 11.4 & 6.94 & 10.05 & 141.4 & 59 \\
L4O & 14 & 14.2 & 6.48 & 10.01 & 51.7 & 21 \\
L4P & 16 & 13.6 & 6.63 & 10.55 & 67.8 & 28 \\
L4V & 20 & 16.7 & 6.76 & 9.6 & 80 & 32 \\
L4I & 9 & 9.7 & 6.73 & 9.9 & 73.9 & 31 \\
L5O & 16 & 13.9 & 6.79 & 10.57 & 162 & 65 \\
L5P & 23 & 16.5 & 6.79 & 9.3 & 150.9 & 61 \\
L5V & 22 & 19.1 & 6.91 & 9.1 & 186 & 74 \\
L5I & 14 & 12.1 & 6.91 & 9.94 & 170.7 & 71 \\
L6O & 15 & 14.9 & 6.66 & 9.76 & 93.7 & 38 \\
L6P & 18 & 14.6 & 6.69 & 10.48 & 111.8 & 46 \\
L6V & 22 & 16.3 & 6.5 & 10.2 & 123 & 49 \\
L6I & 9.5 & 11.3 & 6.55 & 9.78 & 118.7 & 49
\end{tabular}

\begin{tabular}{|c|c|c|c|c|c|c|}
\hline Muestras & $\mathrm{T}^{\circ}$ aire & $\mathrm{T}^{\circ}$ agua & $\mathrm{pH}$ & $\mathrm{O}_{2}(\mathrm{mg} / \mathrm{l})$ & conductividad & SST \\
\hline $\mathrm{L} 7 \mathrm{O}$ & 17 & 15.6 & 7.94 & 9.9 & 137.2 & 56 \\
\hline L7P & 21 & 17.3 & 6.8 & 9.2 & 151.9 & 62 \\
\hline L7V & 20 & 19 & 6.9 & 8.8 & 191 & 77 \\
\hline L7I & 15 & 11.5 & 6.88 & 10.19 & 70.6 & 71 \\
\hline $\mathrm{L} 8 \mathrm{O}$ & 15 & 14.3 & 7.07 & 9.62 & 193 & 77 \\
\hline L8P & 19 & 16.5 & 6.93 & 9.63 & 179.6 & 73 \\
\hline L8V & 21 & 19 & 6.89 & 8.7 & 156 & 62 \\
\hline L8I & 16 & 12.1 & 7.03 & 9.8 & 188 & 78 \\
\hline L9O & 17 & 14.3 & 6.66 & 9.65 & 160 & 64 \\
\hline L9P & 21 & 15.9 & 6.43 & 8.6 & 154 & 62 \\
\hline L9V & 22 & 18.2 & 6.65 & 9.3 & 150 & 60 \\
\hline L9I & 15.5 & 12 & 6.67 & 9.96 & 162 & 67 \\
\hline L10O & 18 & 15.1 & 6.75 & 9.68 & 66.2 & 27 \\
\hline L10P & 19 & 14.1 & 5.4 & 9.86 & 63.4 & 26 \\
\hline L10V & 24 & 17.3 & 5.73 & 7.5 & 85 & 32 \\
\hline L10I & 14 & 11.6 & 6.5 & 10.25 & 78.5 & 32 \\
\hline M1O & 15 & 14.4 & 9.32 & 9.29 & 34 & 14 \\
\hline M1P & 21 & 13.8 & 5.79 & 8.55 & 53 & 21 \\
\hline M1V & 21 & 15.7 & 5.3 & 9.4 & 56 & 23 \\
\hline M1I & 9 & 11.4 & 5.95 & 8.81 & 36.6 & 21 \\
\hline $\mathrm{M} 2 \mathrm{O}$ & 17 & 15 & 7.58 & 9.92 & 70 & 28 \\
\hline $\mathrm{M} 2 \mathrm{P}$ & 19 & 16.2 & 7.02 & 9.25 & 75 & 30 \\
\hline M2V & 27 & 17.4 & 6.3 & 9.6 & 81 & 32 \\
\hline M2I & 8.5 & 11.8 & 6.5 & 9.81 & 64.4 & 36 \\
\hline M3O & 15 & 14 & 7.07 & 9.46 & 39 & 16 \\
\hline M3P & 21 & 17.6 & 7.37 & 9.2 & 39.5 & 10 \\
\hline M3V & 20 & 16.3 & 5.89 & 10.6 & 21 & 52 \\
\hline M3I & 16 & 12.2 & 6.54 & 11.12 & 31.3 & 17 \\
\hline M4O & 19 & 14.9 & 6.28 & 7.6 & 40.4 & 16 \\
\hline M4P & 19 & 14.6 & 5.5 & 8.26 & 45 & 18 \\
\hline M4V & 20 & 13.6 & 5.02 & 8.9 & 55 & 22 \\
\hline M4I & 11 & 11.3 & 5.48 & 8.46 & 33.3 & 19 \\
\hline M5O & 16 & 14.7 & 6.8 & 10.26 & 28.15 & 11.5 \\
\hline M5P & 24 & 15.6 & 6.47 & 9.52 & 42.5 & 17 \\
\hline M5V & 19 & 16.2 & 5.78 & 11.3 & 44 & 17 \\
\hline M5I & 12 & 11.8 & 6.44 & 9.95 & 30.7 & 17 \\
\hline M60 & 16 & 14.9 & 6.14 & 9.74 & 30.5 & 12 \\
\hline M6P & 19 & 17.2 & 7.45 & 8.8 & 33.5 & 14 \\
\hline M6V & 20 & 15.8 & 5.54 & 10 & 44 & 17 \\
\hline M6I & 12 & 11.6 & 6.18 & 10.03 & 22.7 & 13 \\
\hline M7O & 18 & 14.8 & 6.73 & 10.05 & 61 & 24 \\
\hline M7P & 29 & 17.7 & 6.45 & 8.71 & 62 & 25 \\
\hline M7V & 22 & 17.8 & 6.51 & 8.6 & 82 & 33 \\
\hline M7I & 17 & 12.7 & 6.17 & 9.95 & 48.6 & 27 \\
\hline M8O & 18 & 14.5 & 6.73 & 10.04 & 58 & 23 \\
\hline M8P & 24 & 17.8 & 6.44 & 8.92 & 58 & 23 \\
\hline M8V & 21 & 17.3 & 6.46 & 9.7 & 69 & 28 \\
\hline M8I & 16 & 12.7 & 6.57 & 10.81 & 42.5 & 23 \\
\hline M9O & 16 & 15.3 & 7.78 & 9.88 & 37.6 & 14 \\
\hline M9P & 22 & 16.7 & 6.61 & 9.4 & 54 & 22 \\
\hline M9V & 20 & 17 & 5.98 & 10.4 & 80 & 32 \\
\hline M9I & 15 & 12.6 & 6.59 & 10.04 & 50 & 27 \\
\hline M10O & 15 & 14.2 & 6.19 & 10.07 & 30.1 & 12 \\
\hline M10P & 24 & 14.8 & 6.83 & 9.7 & 35 & 14 \\
\hline M10V & 19 & 15.7 & 5.63 & 10.8 & 38 & 15 \\
\hline M10I & 13 & 12.1 & 6.5 & 9.81 & 27.1 & 15 \\
\hline
\end{tabular}




\section{Variables ambientales}

En la Tabla 3 se relacionan los parámetros físicos y químicos medidos en las distintas campañas de muestreo en ambos ríos (L: Lagares, M: Miñor) y en las cuatro épocas estacionales. Los datos revelan una posible alteración provocada por contaminación moderada a fuerte en el río Lagares y leve a moderada en el río Miñor.

Se puede observar que la mayoría de las variables presentan un rango de valores amplio (en porcentaje de concentración) aunque, prácticamente en todos los casos los valores más bajos corresponden al río Miñor y los más altos al río Lagares. De una forma general los valores observados en el río Lagares son muy superiores a los observados en el río Miñor sobre todo para el oxigeno disuelto, $\mathrm{pH}$, conductividad y SST.

$\mathrm{Si}$ comparamos los valores de las variables analizadas con el grado de actividad antropogénica desarrollada en los márgenes de los ríos estudiados (Tabla 1), podemos concluir que justamente los puntos de muestreo cuyas variables presentan valores más altos corresponden a los que consideramos de actividades marginales moderadas o intensas, por lo tanto estos valores elevados de las variables pueden ser un indicio de contaminación. Estos puntos se correspondían con L3, L5, L7, L8 y L9 en la cuenca del río Lagares y con M2, M7, y M9 en la del río Miñor.

\section{Parámetros ecológicos de la comunidad}

En la Tabla 4 se recogen en cada uno de los puntos, los valores medios del año de los parámetros ecológicos de la comunidad. La riqueza total de la cuenca del río Lagares se elevó a 17 especies y en la cuenca del río Miñor casi alcanzó el doble de especies, contabilizándose hasta 31. Si tenemos en cuenta la variable estacional, la riqueza máxima se observó en el punto M4, en primavera, con 13 especies y la mínima fue nula en 16 muestreos, la mayoría de ellos correspondientes al río Lagares. La media general de la riqueza fue de 3.09 especies/muestreo. Los valores totales de abundancia oscilan entre el máximo de 241 ejemplares, recogidos también en el punto M4 en
Tabla 4. Valores medios de riqueza, abundancia y diversidad por puntos de muestreo. Average values of richness, abundance and diversity by sampling site.

\begin{tabular}{lccc}
\hline Muestras & Riqueza & Abundancia & Diversidad \\
\hline L1 & 0.50 & 0.50 & 0.00 \\
L2 & 0.25 & 0.25 & 0.00 \\
L3 & 1.00 & 2.00 & 0.14 \\
L4 & 2.75 & 8.25 & 0.83 \\
L5 & 2.25 & 6.75 & 0.50 \\
L6 & 2.25 & 27.75 & 0.64 \\
L7 & 2.00 & 27.50 & 0.17 \\
L8 & 0.25 & 0.50 & 0.00 \\
L9 & 1.75 & 8.75 & 0.17 \\
L10 & 1.50 & 1.75 & 0.26 \\
M1 & 4.75 & 14.50 & 1.12 \\
M2 & 2.50 & 6.75 & 0.74 \\
M3 & 6.25 & 28.50 & 1.40 \\
M4 & 8.00 & 102.00 & 1.52 \\
M5 & 5.00 & 25.50 & 1.03 \\
M6 & 1.75 & 7.25 & 0.42 \\
M7 & 3.00 & 14.00 & 0.83 \\
M8 & 6.25 & 78.00 & 1.17 \\
M9 & 4.00 & 18.25 & 0.78 \\
M10 & 5.75 & 20.50 & 1.56 \\
\hline
\end{tabular}

primavera, y el mínimo nulo en los 16 muestreos citados anteriormente. La media general fue de 19.96 individuos/muestreo. Los valores de diversidad varían entre el máximo de 2.17 (M4, primavera) y el mínimo nulo en 31 muestreos.

Espacialmente el punto de mayor riqueza fue el M4, con 15 especies y los de menor riqueza L2 y L8, con solamente una especie registrada. El punto con mayor valor de abundancia fue también el M4 con 408 individuos en las 4 campañas, y el menos abundante el L2 con solo 1 individuo recogido en todas las campañas. Los puntos de mayor diversidad media fueron el M10 (1.56) y el M4 (1.52). Por estaciones del año, las épocas con mayor riqueza de coleópteros fueron primavera y verano con 27 especies, y las más abundantes, primavera (676 individuos) e invierno (432 individuos). La mayor diversidad media fue observada en primavera (0.95), seguida por la de verano (0.74).

En la figura 2 se observa la variación estacional de la riqueza, abundancia y diversidad en ambas cuencas hidrográficas. Se puede constatar que las tres variables en ambas cuencas presentan curvas semejantes, en las que se observan que los 


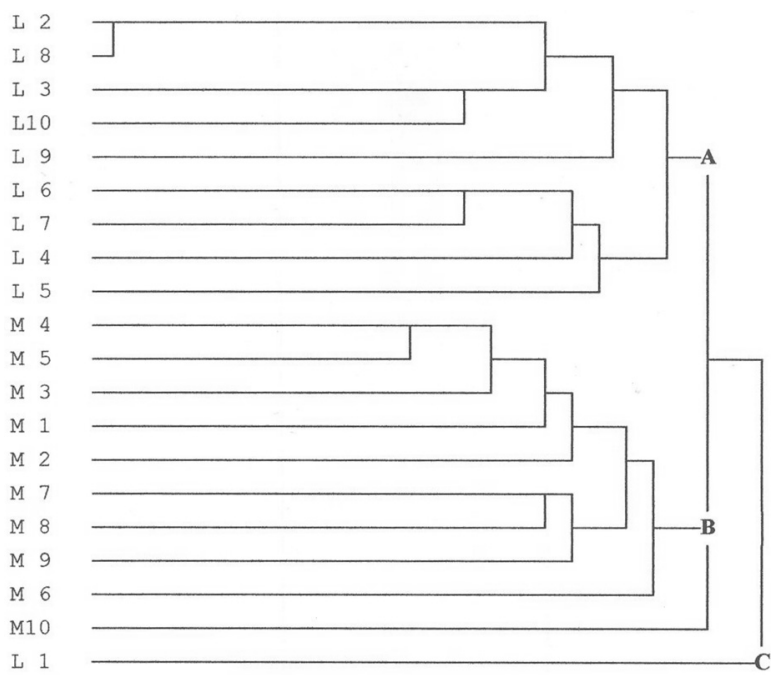

Figura 2. Variación estacional de la riqueza (a), abundancia (b) y diversidad (c) en los ríos Lagares y Miñor. Seasonal variation of the richness (a), abundance $(b)$ and diversity $(c)$ in Lagares and Miñor rivers.

valores más altos corresponden a primavera. Estos valores descienden en verano y alcanzan los valores más bajos en otoño, recuperándose ligeramente en invierno. En todos los casos se observan, en comparación con el río Lagares, valores superiores para el río Miñor, una vez más indicando una mejor calidad biológica en este río.

\section{Afinidad faunística entre estaciones de muestreo}

En relación con la afinidad faunística que presentan los diferentes puntos de muestreo, la figura 3 nos indica la formación de tres grupos claramente separados y con una afinidad faunística muy baja entre ellos (14\%). Los grupos A y B quedan separados claramente entre los del ríos Lagares (grupo A) y del río Miñor (grupo B). El grupo C está formado únicamente por el punto L1 y presenta una afinidad faunística con los demás grupos de solamente $5 \%$.

\section{DISCUSIÓN}

En los estudios ecológicos es importante tener en cuenta la frecuencia de las especies en cada a) Riqueza

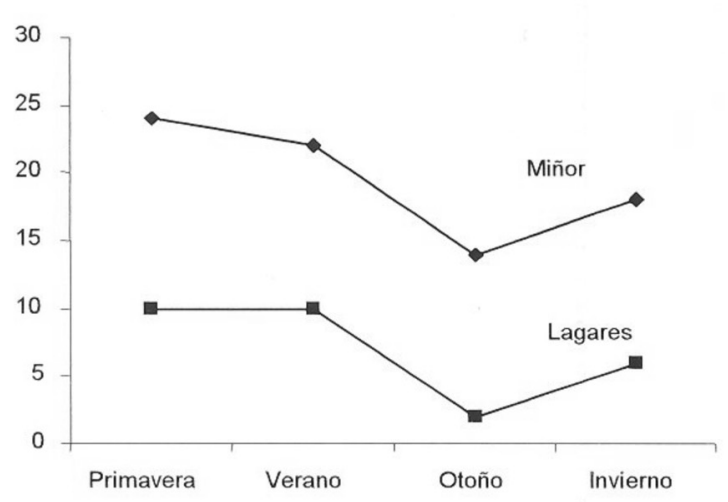

b) Abundancia

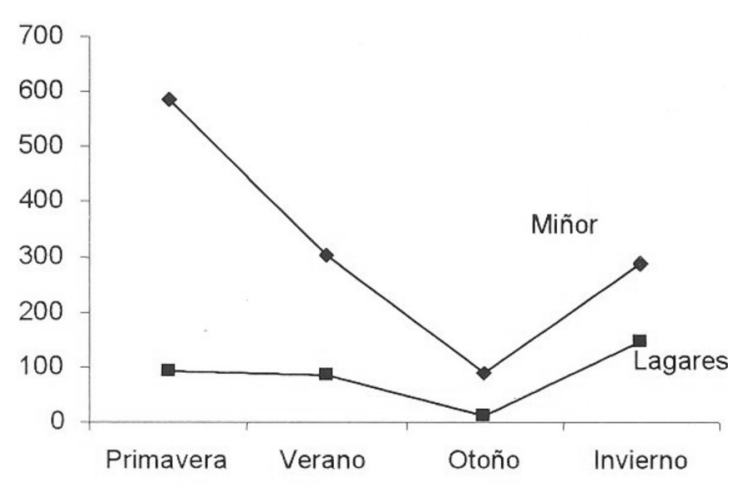

c) Diversidad

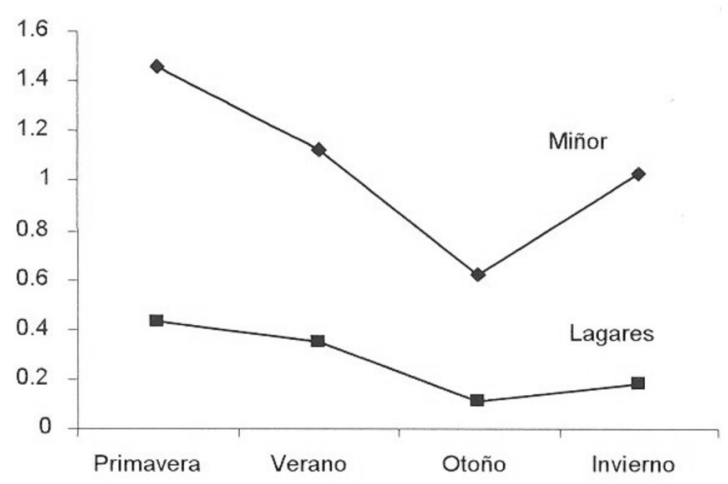

Figura 3. Afinidad faunística entre puntos. Fauna similarity between sites. 
uno de los puntos de muestreo, ya que esto podría ser un indicativo de la calidad de las aguas de un ecosistema, puesto que cuanto más frecuente es una especie, podría implicar que más adaptada esta a ese ambiente en concreto, tal como se expresa en diferentes patrones de dispersión presentados por coleópteros de aguas corrientes, especialmente Elmidae e Hydraenidae (White et al., 1984). En este contexto, hay que señalar que Fernández Díaz (2003) en un estudio de la fauna de coleópteros acuáticos del río Ávia, recoge el $37 \%$ de las especies en más del $45 \%$ de las muestras, contrastando con los datos de este trabajo, donde observamos que la gran mayoría de las especies tuvo una frecuencia inferior al $40 \%$, aunque esas diferencias pueden deberse a la metodología de muestreo empleada en el presente estudio, ya que podría ser considerada por algunos autores como insuficiente, para un muestreo lo más cercano a la distribución natural. Del total de especies, solamente Limnius volkmari fue muy frecuente, siendo una especie relativamente abundante en casi todos los ambientes, aunque predominante en el río Lagares, lo que nos indica que puede ser tolerante a la contaminación.

De entre los parámetros físicos y químicos medidos, la conductividad es uno de los que más afecta a la variación de las comunidades en las aguas continentales. Según Paz (1993) este parámetro es un buen indicador de determinados tipos de polución puesto que los vertidos de aguas residuales suelen traducirse en un aumento de la conductividad. Este parámetro depende del contenido de sales disueltas en el agua (Fernández Aláez et al., 1987; Sabater et al., 1989). Para los ríos gallegos los valores son generalmente bajos y si se detectan valores muy elevados es un indicativo de alteración en la composición del agua (Antelo et al., 1996). En ríos con las mismas características de los aquí estudiados y no contaminados, los valores no suelen sobrepasar los $50 \mu \mathrm{S} / \mathrm{cm}$. Fernández Díaz (2003) obtiene valores medios entre 27.8 y 43.8 para el río Ávia. Por otro lado García Criado (1999) obtiene valores muy altos en puntos afectados por la minería de carbón, muchas veces superiores a $200 \mu \mathrm{S} / \mathrm{cm}$. Para nuestros ríos los valores se presentaron muy divergentes. Los ríos de la cuenca del río Miñor presentaron un valor medio de $47.3 \mu \mathrm{Scm}$, con un pico de $82 \mu \mathrm{Scm}$. En contraposición la cuenca del río Lagares alcanzó un valor medio de $125.2 \mu \mathrm{Scm}$ y un pico de $193 \mu \mathrm{m} / \mathrm{cm}$, lo que puede ser justificado por su gran afección por la contaminación urbana.

En cuanto al SST, en condiciones normales no suele presentar valores muy altos, por encima de $40 \mathrm{mg} / \mathrm{l}$. Los valores obtenidos por Fernández Díaz (2003) para el río Ávia estuvieron comprendidos entre 9 y $23 \mathrm{mg} / \mathrm{l}$. En contrapartida Fernández Aláez et al. (1987) obtiene valores de $60 \mathrm{mg} / \mathrm{l}$ en el curso alto del río Órbigo, indicando cierto grado de contaminación. En el presente trabajo los valores muy superiores de SST presentados por los puntos del río Lagares pueden ser, una vez más, un claro indicativo de contaminación. Hellawell (1986) comenta que la presencia de altos contenidos de sólidos disueltos en el agua tiene importantes efectos químicos y biológicos pues provoca una disminución del oxígeno, aumento de la turbidez, modifica el sustrato y altera la estructura de las comunidades biológicas.

En este estudio, en relación con los parámetros de la comunidad, la riqueza de coleópteros acuáticos (Adephaga y Polyphaga) es considerablemente baja en comparación con la que se ha obtenido en otros estudios semejantes realizados en ríos del norte de España. Así, en la cuenca del río Ávia (Ourense), y también en 20 puntos de muestreo, la riqueza se eleva a 45 especies de coleópteros acuáticos de Adephaga y Polyphaga (Fernández Díaz, 2003). La diferencia es menos significativa si se compara con las 31 especies de la cuenca del río Órbigo en León (García-Criado, 1999) en 22 puntos de muestreo, aunque hay que destacar que en este caso sólo se estudiaron las familias Elmidae y Hydraenidae. Por otro lado en la cuenca del río Landro en Lugo, se contabilizaron 34 especies pertenecientes al suborden Adephaga (Paz, 1993) en 17 puntos de muestreo. Si comparamos los datos de riqueza específica en las dos cuencas estudiadas encontramos una diferencia muy significativa, previsiblemente resultado de la menor calidad de agua presentada por el río Lagares. 
La abundancia también presentó valores muy bajos (1597 individuos) comparados con otros estudios: 6250 individuos en el río Ávia (Fernández Díaz, 2003), 8207 individuos en el río Landro (Paz, 1993) y 4962 individuos en el río Órbigo (García Criado, 1999).

En cuanto a la diversidad, el máximo del índice de Shannon-Wiener fue de 2.17 (punto M4, primavera) y la media general fue de 0.66 según Magurran (1989) los valores habitualmente registrados para ríos no contaminados están comprendidos entre 1.5 a 3.5. Para el área de estudio los valores del índice son muy bajos, especialmente si comparamos con ríos cercanos. en el río Landro (Paz, 1993) el promedio fue de 2.9; en el río Ávia (Fernández Díaz, 2003) el promedio fue 2.39 y en el río Órbigo (García Criado, 1999) los valores estuvieron comprendidos entre 1.64 y 3.16 de acuerdo con Cao et al., (1996) es de esperar que la diversidad disminuya en puntos afectados por la contaminación ya que el efecto esperado es la disminución de un gran número de especies y aumento de la abundancia de unas pocas, tolerantes al impacto. comparando los dos ríos, una vez más encontramos valores muy superiores de este parámetro en el Miñor, indicando su mejor estado de conservación.

La variación estacional de las tres variables en las dos cuencas estudiadas, presentó un comportamiento similar, con los mayores valores obtenidos en primavera, seguido de verano, algo superior a invierno y los menores valores en otoño, en ese caso debido principalmente por las intensas precipitaciones registradas en el período de muestreos, provocando un aumento considerable del caudal y dispersión de las poblaciones. La principal causa de la variación estacional en poblaciones de coleópteros acuáticos es debido a su fenología. es normal esperar que los valores más altos sean registrados en primavera ya que es cuando ocurre una explosión de emergencias en la mayoría de las especies, reflejando así en un mayor número de adultos muestreados (Valladares et al., 1994; Valladares \& Garrido, 2001).

Según el análisis de afinidad faunística los ríos Lagares y Miñor quedan claramente separados en dos grupos y los principales factores que aíslan faunisticamente esos dos ríos son de tipo geográfico (quedan separados por una serie de montañas costeras), el intercambio fluvial nulo y las diferencias en la calidad de sus aguas, reflejado esto último en los valores tan distintos de los parámetros ambientales analizados. Probablemente estas sean algunas de las causas importantes que provoquen una variación faunística tan marcada. quizás la elevada conductividad sea uno de los factores condicionantes de la distribución de especies en estos ríos, ya que puede estar limitando el establecimiento de poblaciones de muchas especies, especialmente en el río Lagares.

Considerando todo lo expuesto y los resultados obtenidos, tanto de tipo faunístico como de tipo ecológico y ambiental, se puede concluir afirmando que la contaminación causada principalmente por factores antropogénicos en las cuencas hidrográficas de los ríos Lagares y Miñor parece evidente. Según se pudo demostrar en este estudio la disminución de la riqueza, abundancia y diversidad en los puntos moderadamente impactados está directamente relacionada con el aumento en los valores de parámetros ambientales como el pH, oxígeno disuelto, conductividad y SST. Estos parámetros aunque puedan presentar variaciones naturales debido a la heterogeneidad de las aguas y que estas variaciones pueden ser consideradas normales, podemos suponer que son indicativos de contaminación ya que, aunque ambos ríos presentan unas características geomorfológicos muy similares, los parámetros ambientales medidos son muy diferentes, tal como quedó demostrado.

Según los datos obtenidos queda claro que los ríos de la cuenca del Miñor presentan una mejor calidad de agua, estando menos contaminados y mejor conservados, además de presentar una mayor diversidad biológica y una comunidad de coleópteros acuáticos más homogénea y mejor estructurada, como se puede constatar por el alto número de endemismos presentes. Sin embargo hay que destacar los valores bajos de parámetros ecológicos obtenidos en ciertos puntos de muestreo de esa cuenca, lo que nos indica que se ven afectados por la presión antropogénica y por ello necesitan ser sometidos a una mejor conservación. 


\section{AGRADECIMIENTOS}

Este estudio se ha beneficiado de la Xunta de Galicia (Consellería de Medio Ambiente), con el proyecto PGIDTOOPXI30122PR. Igualmente, la Agencia Española de Cooperación Internacional (Ministerio de Asuntos Exteriores), ha concedido una ayuda al primer autor para desarrollar su actividad en España dentro de este proyecto.

\section{BIBLIOGRAFÍA}

ABELLÁN, P. D. SÁNCHEZ-FERNÁNDEZ, J. VELASCO \& A. MILLÁN. 2005. Assesing conservation priorities for insects: status of water beetles in southeast Spain. Biological Conservation, 121:79-90.

ANTELO, J. M., F. ARCE \& J. R. CARBALLEIRA. 1996. As características físicoquímicas das augas superficiais. In: As Augas de Galicia. F. DiazFierros (ed.): 352-446. Consello da Cultura Galega, Santiago de Compostela.

ARGERICH, A. M. A. PUIG, \& E. PUPILLI. 2004. Effect of floods of different magnitude on the macroinvertebrate communities of Matarranya stream (Ebro river basin, NE Spain). Limnetica, 23 (3-4): 283-294.

CAO, Y., A. W. BARK \& W. P. WILLIAMS. 1996. Measuring the responses of macroinvertebrate communities to water pollution: a comparison of multivariate approaches, biotic and diversity indices. Hydrobiologia, 341: 1-19.

CASTELLA, E., M. RICHARDOT-COULE, C. ROUX \& P. RICHOUX. 1984. Macroinvertebrates as "describers" of morphological and hydrological types of aquatic ecosystems abandoned by Rhone River. Hydrobiologia, 119: 219-225.

CASTRO A., J. M. HIDALGO y A. M. CÁRDENAS. 2003. Nuevos datos sobre los coleópteros acuáticos del Parque Nacional de Doñana (España): Capturas realizadas mediante trampas de luz y técnicas de muestreo para fauna edáfica. Bol. S.E.A., 33: 153-159.

EYRE, M. D., J. G. PILKINGTON, R. CARR, R. P. MCBLANE, S. P. RUSHTON \& G. N. FOSTER. 1993. The running-water beetles (Coleoptera) of a river catchments in northern England. Hydrobiologia, 264: 33-45.

FERNÁNDEZ ALÁEZ, M. y M. IBAÑEZ y C. FERNÁNDEZ ALÁEZ. 1987. Estudio físico-químico de los ríos de la cuenca del Bernesga (León). Actas del IV Congreso español de limnología, 179-190.

FERNÁNDEZ DÍAZ, M. 2003. Estudio faunístico y ecológico de los coleópteros acuáticos (Adephaga y Polyphaga) en la cuenca del río Ávia (Ourense, NO España): Distribución espacial y temporal. Tesis de licenciatura, Universidad de Vigo. 146 pp. FLECHTNER, G. 1986. Association analysis of water-beetles communities (Coleoptera, Dytiscidae et Haliplidae). Entomologica Basiliensia, 11: 297-308.

FOSTER, G. N., A. P. FOSTER, M. D. EYRE \& D. T. BILTON, 1990. Classification of water beetles assemblages in arable fenland and ranking of sites in relation to conservation value. Freshwat. Biol., 22: 343-354.

FOSTER, G. N., B. H. NELSON, D. T. BILTON, D. A. LOTT, R. MERRIT, R. S. WEYL. \& M. D. EYRE. 1992. A classification and evaluation of Irish water beetle assemblages. Aquatic Conserv: Mar. Freshw. Ecosyst., 2: 185-208.

GALLARDO, A., FRESNEDA, J. y J. TOJA. 1995. Distribución de los coleópteros acuáticos (Insecta, Coleoptera) en dos cuencas del Sur de la Península Ibérica. Relaciones con algunos factores del medio. Limnetica, 11: 19-28.

GARCÍA CRIADO, F. 1999. Impacto de la minería del carbón sobre Hydraenidae y Elmidae (Coleoptera) en la cuenca del río Sil (León, España). Tesis doctoral, Universidad de León. 281 pp.

GARCÍA-CRIADO, F. 2002. Distribución y autoecología de Coleóptera acuáticos en ríos afectados por minería del carbón (cuenca del Sil, León, España). 2. Hydraenidae. Boln. Asoc. Esp. Ent., 26 (1-2): 69-89.

GARCÍA CRIADO, F. y M. FERNÁNDEZ ALÁEZ. 1994. Distribución longitudinal de Hydraenidae y Elmidae (Coleoptera) en la cuenca del río Órbigo (León, España). Orsis, 9: 37-57.

GARCÍA CRIADO, F. y M. FERNÁNDEZ ALÁEZ. 1995. Aquatic Coleoptera (Hydraenidae and Elmidae) as indicators of the chemical characteristics of water in the Órbigo River basin (N-W Spain). Ann. Limnol., 31: 185-199.

GARCIA-CRIADO, F., M. FERNÁNDEZ y J. REGIL. 1995. Datos sobre la ecología de la familia Elmidae en la cuenca del río Orbigo (León, España). Boll. Soc. ent. Ital., 126 (3): 200-210.

GARCÍA-CRIADO, F., C. FERNÁNDEZ ALÁEZ \& M. FERNÁNDEZ-ALÁEZ. 1999. Environmental variables influencing the distribution of Hydrae- 
nidae and Elmidae assemblages (Coleoptera) in a moderately-polluted river basin in north-western Spain. Eur. J. Entomol., 96: 37-44.

GARCÍA CRIADO, F. \& M. FERNÁNDEZ ALÁEZ. 2001. Hydraenidae and Elmidae assemblages (Coleoptera) from Spanish river basin: good indicators of coal mining pollution? Arch. Hydrobiol., 150: 641-660.

GARRIDO J. y J. A. REGIL. 1994. Fauna acuática de la Cordillera Cantábrica. I. El género Hydroporus Clairville, 1806 en la provincia fitogeográfica Orocantábrica (Coleoptera, Dytiscidae). Boll. Soc. ent. ital., 126 (1): 9-30.

GARRIDO, J. y C. E. SÁINZ-CANTERO. 2004. Diversidad de coleópteros acuáticos en la Península de Barbanza (Galicia, NW España) (Coleoptera, Adephaga y Polyphaga). Nouv. Revue Ent. (N.S.), 21: 49-64.

GARRIDO J. y A. GAYOSO, 2005. Elmidae y Dryopidae del Sistema Ibérico (España) (Coleoptera: Byrrhoidea). Bull. Soc. entomol. France, 110 (3): 359-366.

GARRIDO, J., J. A. DÍAZ PAZOS y J. A. RÉGIL CUETO. 1994. Fauna acuática de la Comunidad Foral de Navarra (España) (Col., Adephaga y Polyphaga). Bull. Soc. entomol. France, 99: 131148.

GARRIDO, J., C. E. SAINZ-CANTERO y J. A. DÍAZ PAZOS. 1996. Fauna entomológica del Parque Nacional de Doñana (Huelva, España) I. (Coleoptera, Polyphaga). Nouv. Revue Ent. (N.S.), 13: 57-71.

GARRIDO, J., C. E. SAINZ-CANTERO y J. A. RÉGIL CUETO. 1997. Fauna entomológica del Parque Nacional de Doñana (Huelva, España) II. (Coleoptera, Adephaga). Nouv. Revue Ent. (N.S.), 14: 365-377.

GAYOSO, A., J. M. SANTAMARÍA y J. C. OTERO. 1997. Contribución al conocimiento de la familia Elmidae Curtis, 1830 (Coleoptera, Dryopoidea) en Galicia (NW Península Ibérica). Nova Acta Científica Compostelana (Bioloxía) 7: 193-206.

GONZÁlEZ, J. M., A. BASAGUREN \& J. POZO. 2003. Macroinvertebrate communities a thirdorder Iberian stream. Annales de LimnologieInternational Journal of Limnology, 39: 287-296.

GONZÁLEZ, S. y F. NOVOA. 1988. Estudio faunístico sobre los coleópteros acuáticos de Galicia. I. Dytiscidae. Boln. Asoc. esp. Ent., 12: 59-72.
GONZÁlEZ, S. y F. NOVOA, 1995. Estudio faunístico sobre los coleópteros acuáticos de Galicia. II. Haliplidae, Gyrinidae, Hygrobiidae y Dytiscidae. Boln. Asoc. esp. Ent., 19: 9-21.

GONZÁLEZ, J., NOVOA, F. y A. BASELGA. 2005. Coleópteros acuáticos de la Sierra de Xistral, noroeste de la Península Ibérica (Coleoptera: Gyrinidae, Haliplidae, Noteridae, Dytiscidae e Hydrophilidae). Nouv. Revue Ent., 22 (2): 107-115.

GUERRERO, C. M ${ }^{\mathrm{a}}$ R. VIDAL-ABARCA, $\mathrm{M}^{\mathrm{a}}$ L. SUÁREZ, R. GÓMEZ y M. ORTEGA. 2004. Estructura de tamaño de la comunidad de macroinvertebrados acuáticos de un río de características semiáridas durante el estiaje (río Chimaco, S. E. España). Limnetica, 23 (1-2): 37-56.

HELLAWELL, J. M. 1986. Biological indicators of freshwater pollution and environment management. Elsevier Applied Science Publishers, London. 546 pp.

LOZANO-QUILIS, M. A., A. PUJANTE y F. MARTÍNEZ-LÓPEZ. 2001. Macroinvertebrados y calidad de las aguas de algunos ríos de la provincia de Valencia (España). Bol. R. Soc. Esp. Hist. Nat. (Sec. Biol.), 96: 151-164.

MAGURRAN, A. E. 1989. Diversidad ecológica y su medición. Vedrà, Barcelona. 200 pp.

MILLAN, A., J. VELASCO y SOLER, A. G. 1992. Los coleópteros Hydradephaga de la cuenca del río Segura. SE. de la Península Ibérica. Aspectos faunísticos más relevantes (Coleoptera). Anales de Biología., 18: 39-45.

MILLAN, A., J. VELASCO y A. G. SOLER. 1993. Los coleópteros Hydradephaga de la cuenca del río Segura. (SE. de la Península Ibérica. ). Estudio Corológico. Boln. Asoc. esp. Ent., 17 (1): 19-37.

MILLAN, A., J. VELASCO, SUAREZ, VIDALABARCA y RAMIREZ-DÍAZ. 1996. Distribución espacial de los adephaga acuáticos (Coleoptera) en la cuenca del río Segura (SE. de la Península Ibérica. ). Limnetica 12 (2): 13-29.

MILLAN, A., J. L. MORENO y J. VELASCO, 1997. Coleópteros y Heterópteros acuáticos del complejo Lagunar del río Arquillo (Albacete). Al-Basit. Revista de estudios albacetenses, 40: 29-69.

MILLÁN, A., J. L. MORENO, J. L. y J. VELASCO. 2001a. Estudio faunístico y ecológico de los Coleópteros y Heterópteros acuáticos de las lagunas de Albacete (Alboraj, Los Patos, Ojos de Villaverde, Ontalafia y Pétrola). Sabuco, Revista de Estudios Albacetenses, 1: 43-94. 
MILlÁN, A., MORENO, J. L. y J. VELASCO. 2001b. Estudio faunístico y ecológico de los Coleópteros y Heterópteros acuáticos de las lagunas de Albacete (Lagunas de Ruidera, Salinas de Pinilla, Laguna de Saladar, Laguna del Salobralejo, Lagunas de Corral Rubio, Fuente de Isso y Fuente de Agua Ramos). Sabuco, Revista de Estudios Albacetenses, 2: 167-214.

MILLAN, A., J. L. MORENO y J. VELASCO. 2002. Los coleópteros y heterópteros acuáticos y semiacuáticos de la provincia s Albacete. Catálogo faunístico y estudio ecológico. Instituto de estudios albacetenses "Don Juan Manuel. 180 pp.

MilláN, A., HERNANDO, C., AGUILERA, P., CASTRO, A. e I. RIBERA. 2005. Los coleópteros acuáticos y semiacuáticos de Doñana: reconocimiento de su biodiversidad y prioridades de conservación. Boletín S.E.A., 36: 157-164.

MONTES, C., L. RAMÍREZ y A. G. SOLER, 1982. Variación estacional de las taxocenosis de Odonatos, Coleópteros y Heterópteros acuáticos en algunos ecosistemas del bajo Guadalquivir (SW España) durante un ciclo anual. Anales de la Universidad de Murcia, 38: 21-100.

MORENO, J. L., A. MILLÁN, M. L. SUÁREZ, M. R. VIDAL-ABARCA \& J. VELASCO. 1997. Aquatic Coleoptera and Heteroptera assemblages in waterbodies from ephemeral coastal streams ("ramblas") of south-eastern Spain. Arch. Hydrobiol.,141: 93-107.

MUÑOZ, I. 2003. Macroinvertebrate community structure in an intermittent and a permanent Mediterranean streams (NE Spain). Limnetica, 22(3-4): 107-116.

PAZ, C. 1993. Hydradephaga (Coleoptera) en la cuenca del río Landro (NW Península Ibérica). Estudio faunístico y ecológico. Tesis doctoral, Universidad de Santiago de Compostela. 381 pp.

PUIG, M. A. 1983. Distribución y ecología de las especies de Elmidae (Col.: Dryopoidea) de la cuenca del río Llobregat. Boln. Asoc. esp. Ent., 7: 211-219.

RÉGIL CUETO, J. A. y J. GARRIDO. 1998. Fauna acuática de las Lagunas de Villafáfila (Zamora, España) (Coleoptera, Adephaga). Bull. Soc. entomol. France, 98: 371-380.

RIBERA, I. y G. N. FOSTER. 1993. Uso de coleópteros acuáticos como indicadores biológicos (Coleoptera). Elytron, 6: 61-75.
RIBERA, I. y P. AGUILERA. 1995. Coleópteros acuáticos de la provincia de Huesca (Aragón, España). Zapateri-Revta. aragon. ent., 5: 7-34.

RIBERA, I, J. ISART y J. A. REGIL. 1995a. Autoecología de algunas especies de Hydradephaga (Coleoptera) de los Pirineos. I. Gyrinidae, Haliplidae, Noteridae e Hygrobiidae. Zool. baetica, 6: 33-58.

RIBERA, I., J. ISART \& J.A. REGIL. 1995b. Autoecología de algunas especies de Hydradephaga (Coleoptera) de los Pirineos. II. Dytiscidae. Zool. baetica, 6: 59-104.

RIBERA, I., C. HERNANDO \& P. AGUILERA. 1998. An annotated checklist of the Iberian water beetles (Coleoptera). Zapateri-Revta. aragon. ent., 8: 43-111.

RICO, E. 1996. Distribucción de los Elmidae (Coleoptera: Dryopoidea) en la Península Ibérica e Islas Baleares. Graellsia, 52: 115-147.

RICO, E. \& J. GARCÍA-AVILÉS. 1998. Distribution, autoecology and biogeography of Dryopidae and Elmidae (Coleoptera, Dryopoidea) in the Balearic Islands. Graellsia, 54: 53-59.

RODRÍGUEZ IGLESIAS, F. 1998. Atlas de Galicia. Hércules de Ediciones, A Coruña. 239 pp.

SABATER, F., J. ARMENGOL \& S. SABATER. 1989. Measuring discontinuities in the Ter river. Regul. Rivers: Res. Mgmt., 3: 133-142.

SAINZ-CANTERO, C. E. y J. ALBA TERCEDOR. 1991a. Los Adephaga acuáticos de Sierra Nevada (Granada, España) (Coleoptera: Haliplidae, Gyrinidae, Dytiscidae). Boln. Asoc. esp. Ent., 15: 91109.

SAINZ-CANTERO, C. E. y J. ALBA TERCEDOR. 1991b. Los Polyphaga acuáticos de Sierra Nevada (Granada, España) (Coleoptera: Hydraenidae, Hydrophilidae, Elmidae, Dryopidae). Boln. Asoc. esp. Ent., 15: 171-198.

SAINZ-CANTERO, C. E. y J. L. CORTES-ROMERO, 1996. Coleopterofauna acuática de las $S^{o}$ de Tejeda y Almijara (S. de España). I Adephaga (Col., Haliplidae, Gyrinidae, Dytiscidae). Nouv. Revue. Ent., 13 (3): 249-260.

SÁINZ-CANTERO, C. E., A. SÁNCHEZ ORTEGA y J. ALBA TERCEDOR. 1987. Distribución y autoecología de Hydraenidae (Col.) en Sierra Nevada (España). Boln. Asoc. esp. Ent., 11: 355-365.

SÁNCHEZ-FERNÁNDEZ, D., P. ABELLÁN, J. VELASCO y A. MILLÁN, 2003. Los coleópteros acuáticos de la Región de Murcia. Catálogo faunístico y áreas prioritarias de conservación. Monografías SEA, $\mathrm{n}^{\circ} 10.71 \mathrm{pp}$. 
SÁNCHEZ-FERNÁNDEZ D., P. ABELLÁN, J. VELASCO y A. MILLÁN. 2004. Areas prioritarias de conservación en la cuenca del río Segura utilizando los coleópteros acuáticos como indicadores. Limnetica, 23 (3-4): 209-228.

VALLADARES, L. F. y J. GARRIDO. 2001. Coleópteros acuáticos de los humedales asociados al Canal de Castilla (Palencia, España): Aspectos faunísticos y fenológicos (Coleoptera, Adephaga y Polyphaga). Nouv. Revue Ent. (N.S.), 18 (1): 61-76.

VALLADARES, L. F., J. GARRIDO \& B. HERRERO. 1994. The annual cycle of the community of aquatic Coleoptera (Adephaga and Polyphaga) in a rehabilitated wetland pond: the Laguna de La Nava (Palencia, Spain). Annls Limnol., 30 (3): 209-220.
VALLADARES, L. F., J. DIAZ y J. GARRIDO, 2000. Coleópteros acuáticos del Sistema Ibérico Septentrional (Coleoptera: Haliplidae, Gyrinidae, Dytiscidae, Hydraenidae, Helophoridae, Hydrochidae, Hydrophilidae). Boln. Asoc. esp. Ent., 24 (3-4): 59-84.

VALLADARES, L. F., J. GARRIDO \& F GARCÍACRIADO, 2002. The assemblages of aquatic coleoptera from shallow lakes in the northern Iberian Meseta: Influence of environmental variables. Eur. J. Entomol., 99: 289-298.

WHITE, D. S., W. U. BRIGHAM \& J. T. DOYEN. 1984. Aquatic Coleoptera. In: An Introduction to the aquatic insects of North America.R. W. Merritt \& K. W. Cummins. (eds.): 361-437. Kendall-Hunt Pub. Co., Dubuque. 